\title{
CONDUCTIVIDAD HIDRÁULICA EN MONOLITOS DE SUELO DE CHILE CENTRAL
}

\author{
Hydraulic Conductivity on Soil Monoliths of Central Chile
}

\author{
Manuel Casanova P.*, Osvaldo Salazar G., Oscar Seguel S., Verónica Noguer A. \\ Departamento de Ingeniería y Suelos, Facultad de Ciencias Agronómicas, Universidad de Chile. \\ *Corresponding author: mcasanov@uchile.cl
}

\begin{abstract}
Four monoliths with contrasting textural classes $\left(\mathrm{S}_{\mathrm{c}}, \mathrm{S}_{\mathrm{cl}}, \mathrm{S}_{\mathrm{sl}}\right.$ and $\left.\mathrm{S}_{\mathrm{ls}}\right)$ from soils (Fluventic Haploxerolls and Typic Xerochrepts) of central Chile were used to obtain soil hydraulic conductivity at different slope gradients $(0 \%, 15 \%, 20 \%$ and $25 \%)$ with tension infiltrometer, under laboratory conditions. A total of 13 supply pressure heads ( $\psi_{\mathrm{s}}$, between -140 and $-20 \mathrm{~mm}$ ) were used to obtain the unsaturated hydraulic conductivity $\left(\mathrm{K}_{\psi}\right)$. Paired data $\left[\ln \left(\mathrm{K}_{\psi}\right)\right.$ y $\left.\psi_{s}\right]$ were adjusted by two-linear models that assumed a continuous exponential function in a macroporous and mesoporous system, which considered a boundary supply pressure head. Extrapolating to zero supply pressure head, field saturated hydraulic conductivity $\left(\mathrm{K}_{f s}\right)$ was estimated. In all soils, when a gravitational adjustment to supply pressure heads was used, $\mathrm{K}_{f s}$ values were strongly negatively correlated to slope gradient. It is attributed to a refraction of water flow, which considered the greater frictional or viscous resistance generated by inclination.
\end{abstract}

Keywords: hydraulic conductivity, soil monolith, slope gradient, soil texture, tension infiltrometer.

\section{RESUMEN}

Se evaluó, con tensioinfiltrómetro y en condiciones de laboratorio, la conductividad hidráulica de suelos en distintos gradientes de pendiente $(0 \%, 15 \%, 20 \%$ y $25 \%)$ utilizando cuatro monolitos de clases texturales contrastantes $\left(\mathrm{S}_{\mathrm{A}}, \mathrm{S}_{\mathrm{FA}}, \mathrm{S}_{\mathrm{Fa}}\right.$ y $\left.\mathrm{S}_{\mathrm{aF}}\right)$, provenientes de suelos (Fluventic Haploxerolls y Typic Xerochrepts) de la zona central de Chile. Se emplearon 13 potenciales de suministro de agua $\left(\psi_{\mathrm{s}}\right)$ entre -140 y $-20 \mathrm{~mm}$. Obtenida la conductividad hidráulica insaturada $\left(\mathrm{K}_{\psi}\right)$ para cada $\psi_{\mathrm{s}}$, los valores pareados $\left[\ln \left(\mathrm{K}_{\psi}\right)\right.$ y $\left.\psi_{s}\right]$ fueron ajustados a un modelo bilineal, asumiendo que una función exponencial continua de $\mathrm{K}_{\psi}$ es aplicable al sistema macroporoso y mesoporoso, con un quiebre a un potencial límite. La conductividad hidráulica saturada $\left(\mathrm{K}_{\mathrm{fs}}\right)$ se estimó extrapolando al potencial de suministro cero. Así, al realizar un ajuste gravitacional del potencial de suministro, $\mathrm{K}_{f s}$ mostró una marcada tendencia a disminuir en todos los suelos al aumentar el gradiente de pendiente, lo que se atribuye a una refracción del flujo de agua, considerando la mayor resistencia friccional o viscosa que se genera al inclinar el suelo.

Palabras Claves: conductividad hidráulica, monolito de suelo, gradiente de pendiente, textura de suelo, tensioinfiltrómetro 


\section{INTRODUCCION}

La hidrología de laderas constituye un gran desafío, debido a los numerosos procesos que interactúan en estas condiciones y que contribuyen significativamente a la complejidad del sistema.

La posibilidad de establecer una teoría general se ve obstaculizada por la dificultad de cuantificar muchos de estos procesos. Las interacciones entre la posición en el paisaje, las características de las precipitaciones y el uso del suelo, inducen cambios en las características superficiales del suelo, que son determinantes no sólo de la tasa de ingreso de agua al perfil, sino también de la magnitud de la escorrentía superficial (Chahinian et al., 2006; Strudley et al., 2008). En estas condiciones, el entendimiento de ambos procesos resulta crítico en aspectos como diseño de sistemas de riego y drenaje, la recarga de acuíferos y la migración de químicos (nutrientes, plaguicidas $\mathrm{u}$ otros) en el suelo; en modelos de erosión, es esencial para las estrategias de control de este tipo de degradación de los suelos.

El movimiento del agua a través del suelo es un proceso que responde directamente al gradiente hidráulico, e inversamente a la resistencia que opone este medio. De este modo, conceptualmente el inverso de la resistencia, la conductividad hidráulica $(\mathrm{K})$, es una expresión de la habilidad intrínseca de éste para transmitir agua en diversas condiciones. De acuerdo a varios autores (Hillel, 2004; Miyazaki, 2006), la $\mathrm{K}$ presenta magnitudes diversas que conjugan los efectos de las características de la matriz del suelo (porosidad total, distribución de tamaño de poros $\mathrm{y}$ tortuosidad), así como aquellas propias de la solución que se desplaza a través de éste (densidad y viscosidad).
El hecho que la $\mathrm{K}$ del suelo se exprese normalmente bajo unidades de velocidad $\left[\begin{array}{ll}\mathrm{L} & \mathrm{T}^{-1}\end{array}\right]$, la convierte en una variable fácilmente comparable con procesos relacionados como la intensidad de precipitación, u otras de movimiento de agua (Reynolds et al., 2002). La conductividad hidráulica de un suelo saturado $\left(\mathrm{K}_{\mathrm{fs}}\right)$ de estructura estable es en principio constante en el tiempo, ya que se asume que la totalidad del espacio poroso se encuentra colmado del fluido. Sin embargo, en la realidad ciertas variaciones determinan el uso del sufijo fs, el cual denota que la variable se mide en condiciones de campo y acusa la probable ocurrencia de poros con aire atrapado $u$ otros rasgos de difícil control en estas condiciones (Reynolds, 1993).

Considerando que la conductividad hidráulica en medios no saturados $\left(\mathrm{K}_{\psi}\right)$ varía en respuesta a al potencial hídrico $\left(\psi_{s}\right)$ total existente, en el corto plazo de un evento de precipitación los procesos de infiltración y escorrentía son controlados fundamentalmente por la $\left(\mathrm{K}_{\psi}\right)$ del suelo, pero en el largo plazo, dependen particularmente de esta propiedad en condiciones muy cercanas a la saturación (D'Odorico and Porporato, 2006).

Bajo condiciones de suelos estables y homogéneos, se espera que la infiltración decrezca en la medida que el gradiente de pendiente aumente (Zaslavsky and Sinai, 1981). Sin embargo, se advierte en la literatura observaciones contradictorias en cuanto a la influencia de este factor en la tasa de infiltración o en la $\mathrm{K}$ de los suelos, dado que normalmente se ha evaluado comparando mediciones en distintas posiciones de laderas (Mertens et al., 2002; Janeau et al., 2003; Bodhinayake et al., 2004; Bodner et al., 2008). Si se considera el sellamiento que se verifica en las posiciones inferiores, ello explicaría los resultados tanto de López et al. (2003) como de Bradford and Huang (1992) en 
el sentido que la $\mathrm{K}$ es directamente proporcional al gradiente de pendiente.

El tensioinfiltrómetro es un infiltrómetro de disco a tensión, de carga constante, que puede operar bajo potenciales hídricos $\left(\psi_{s}\right)$ diversos, de manera que pueden emplearse para la determinación tanto de propiedades hidráulicas en condiciones saturadas o insaturadas. Si bien hace una década es utilizado en Chile (Casanova et al., 2000a; Casanova et al., 2000b; Casanova et al., 2003; Verbist et al., 2009), ha sido inicialmente descrito en detalle por varios autores (Ankeny et al., 1991; AnguloJaramillo et al., 2000). Entre muchas de sus ventajas, comparado con otros métodos, es simple de usar, genera un mínimo de alteración del sitio, mide la $\mathrm{K}$ cercana a saturación con potenciales controlados $\left(\psi_{s}\right) \mathrm{y}$, consecuentemente, permite caracterizar la transmisión de agua a nivel de los macro y mesoporos del suelo. No obstante, debe ser calibrado y nivelado apropiadamente para su uso, pues la gravedad determina que se generen $\psi_{s}$ distintos al controlado, tanto en la parte alta como en la baja del disco en contacto con el suelo (AnguloJaramillo et al., 2000; Casanova et al., 2000a; Walker et al., 2006). El empleo de arena fina como material de contacto permite subsanar la situación ante superficies rugosas $u$ onduladas, pero en laderas inclinadas algunos autores (Sullivan et al., 1996; Reynolds, 2006) recomiendan alterar el sitio de medición, lo que resulta inapropiado en estudios de impacto del encostramiento y sellamiento en la tasa de infiltración.

Cuando se estudia la compleja hidrología de laderas, resulta conveniente aislar algunos mecanismos y explorarlos separadamente bajo ciertos supuestos que simplifican el sistema (Ridolfi et al., 2003). En esta perspectiva, el objetivo de esta investigación se orientó a evaluar la conductividad hidráulica insaturada $\left(\mathrm{K}_{\psi}\right)$ y estimar la conductividad hidráulica saturada $\left(\mathrm{K}_{\mathrm{fs}}\right)$, en condiciones de laboratorio, empleando un infiltrómetro de disco sobre cuatro monolitos de suelo no disturbado, con clases texturales contrastantes y artificialmente inclinados.

\section{MATERIALES Y METODOS}

En dos áreas localizadas en la Región Metropolitana (coordenadas son $33^{\circ} 26^{\prime} \mathrm{S}$ $-70^{\circ} 49^{\prime} \mathrm{W}$ y $\left.33^{\circ} 28^{\prime} \mathrm{S}-70^{\circ} 50^{\prime} \mathrm{W}\right)$, se extrajeron 4 monolitos cilíndricos de suelos $(0,6 \mathrm{~m}$ de diámetro y $0,45 \mathrm{~m}$ de altura) con clases texturales superficiales contrastantes: arcillosa $\left(\mathrm{S}_{\mathrm{A}}\right)$, franco arcillosa $\left(\mathrm{S}_{\mathrm{FA}}\right)$, franco arenosa $\left(\mathrm{S}_{\mathrm{Fa}}\right) \mathrm{y}$ areno francosa $\left(\mathrm{S}_{\mathrm{aF}}\right)$. Los dos primeros suelos corresponden a Fluventic Haploxerolls y los restantes a Typic Xerochrepts (CIREN, 1996).

De acuerdo a la morfología del perfil de suelo, en cada sitio y de cada horizonte genético se extrajeron muestras en triplicado. El contenido de materia orgánica superficial se calculó mediante calcinación a $550^{\circ} \mathrm{C}$ (Sadzawka et al., 2007), mientras que la textura se determinó con hidrómetro de Bouyoucos (Gee and Bauder, 1986). Finalmente, de acuerdo a la metodología de Klute (1986), la densidad aparente seca se calculó con cilindro, la densidad de partículas con picnómetros y la retención de agua a (33 y $1,500 \mathrm{kPa})$ con plato y olla a presión.

La extracción de monolitos se realizó haciendo ingresar libremente un tambor metálico en el suelo, excavando alrededor sin disturbar el interior. Una vez que la superficie del suelo alcanzó su extremo cerrado, se retiró el bloque con material en exceso y se transportó invertido al laboratorio; allí se eliminó dicho exceso del bloque y se cubrió el fondo con una malla metálica fina. Luego, se invirtió 
sobre una estructura de madera, que posibilitó inclinarlos a cuatro gradientes de pendiente $(0,15,20$ y $25 \%)$ (Figura 1$)$. Finalmente, se decapitó el extremo cerrado del tambor, quedando el horizonte superficial expuesto. Una metodología similar es informada por Cook (2006), aunque para bloques de suelo más pequeños. En consideración a que casi un $60 \%$ de territorio nacional presenta laderas con gradientes de pendiente superiores a un $8 \%$ (FAO, 2000), se estima que los gradientes elegidos son frecuentes en la zona central de Chile.
Para la determinación de la infiltración y la estimación de la conductividad hidráulica en condiciones insaturadas, se utilizó un tensioinfiltrómetro (CSIRO Permeameter, A.L. Franklin Precision Engineers) de $0.20 \mathrm{~m}$ de diámetro y agua destilada. Se garantizó un buen contacto entre la membrana porosa del disco y el suelo, empleando una capa delgada de arena fina en la superficie de cada bloque de suelo. La tasa de infiltración se midió cada 30 segundos y durante 45 min en total, asumiendo que en este tiempo se ha alcanzado el estado de equilibrio.
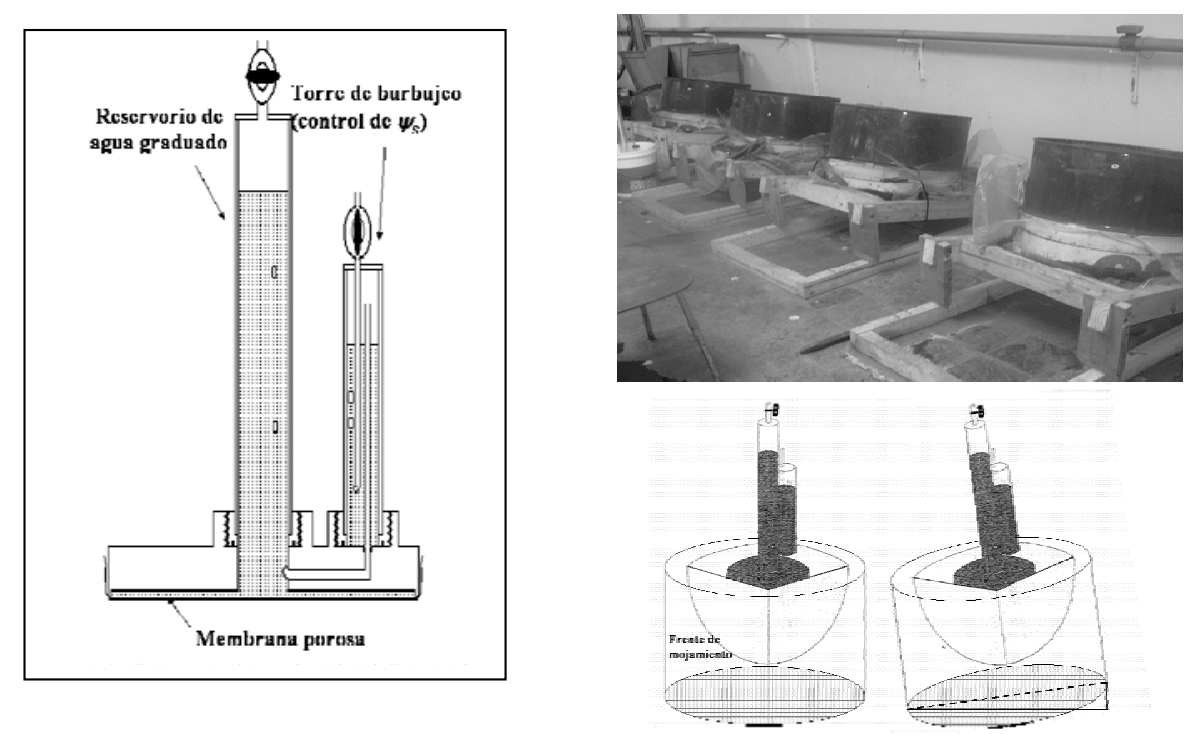

Figura 1. Infiltrómetro de disco a tensión (tensioinfiltrómetro) y sistema de inclinación de monolitos.

Figure 1. Tension disc infiltrometers (tension infiltrometer) and monoliths sloping system.

Se privilegió el empleo de 13 potenciales controlados en la torre de burbujeo $\left(\psi_{s}\right)$ en cada monolito para definir la forma en que se relacionan $\mathrm{K}$ y $\psi_{s}$, más que efectuar repeticiones. Así, las tensiones empleadas fluctuaron entre 140 y $20 \mathrm{~mm}$ a intervalos regulares, y se aplicaron en forma descendente para minimizar el efecto histéresis.

De las numerosas técnicas para analizar los datos de tensioinfiltrómetro $\left(\mathrm{K}_{\psi}, \psi_{s}\right)$, tanto Logsdon and Jaynes (1993) 
como Hussen and Warrick (1993) sugieren las regresiones no lineales basadas en una solución para la infiltración desde una fuente circular (Wooding, 1968), las que entregan resultados rápidos, estables y sin valores negativos. En este sentido, $\mathrm{Hu}$ et al. (2009) confirman que este tipo procedimiento no requiere el conocimiento del contenido de agua, por tanto son útiles para caracterizar la variabilidad espacial y temporal de las propiedades hidráulicas del suelo. Por consiguiente, el análisis de la información se realizó siguiendo la metodología propuesta por Messing and Jarvis (1993), que considera a Wooding (1968) y Ankeny et al. (1991), obteniéndose las conductividades hidráulicas no saturadas $\left(\mathrm{K}_{\psi}, \mathrm{LT}^{-1}\right)$ para cada potencial controlado $\left(\psi_{\mathrm{s}}, \mathrm{L}\right)$. Así estos valores pareados $\left(\mathrm{K}_{\psi}\right.$ ,$\left.\psi_{s}\right)$ se ajustaron a modelos bilineales de regresión con el software NLREG (Sherrod, 2005) asumiendo que una función exponencial continua de $\mathrm{K}_{\psi}$ es aplicable a dos sistemas porosos (macroporos y mesoporos), con una división a una tensión límite $\left(\psi_{b}\right)$ como pivote (Keng and Lin, 1982):

$$
\begin{aligned}
& \ln \mathrm{K}_{\psi}=\ln \mathrm{K}_{b}+\alpha_{\mathrm{M}}\left(\psi_{s}-\psi_{b}\right), \text { si } \psi_{s}>\psi_{b} \\
& \ln \mathrm{K}_{\psi}=\ln \mathrm{K}_{b}+\alpha_{\mathrm{m}}\left(\psi_{s}-\psi_{b}\right), \text { si } \psi_{s} \leq \psi_{b}
\end{aligned}
$$

donde $\alpha_{\mathrm{M}}$ y $\alpha_{\mathrm{m}}$ son las respectivas pendientes de las líneas en la región de macroporos y mesoporos (Luxmoore, 1981) y $K_{b}$ representa el valor de la conductividad hidráulica insaturada en $\psi_{b}$ (Figura 2). La conductividad hidráulica saturada $\left(\mathrm{K}_{\mathrm{fs}}\right)$ se estimó por extrapolación a la tensión o potencial cero.

Teniendo en cuenta que en un suelo no saturado el gradiente del potencial hidráulico está dado principalmente por la suma del potencial gravitacional y el potencial mátrico, se han propuesto dos metodologías de ajuste para mediciones con tensioinfiltrómetros en pendiente (Casanova et al., 2000a). La primera, al observar flujo superficial de agua bajo el disco, en que se asume que a la tensión controlada que se verifica dicho flujo, la tensión efectiva es cero (saturación) y por ende se modifican las tensiones preestablecidas. La segunda, sin flujo superficial bajo el disco, considera que el potencial gravitacional variará entre la parte superior del disco (pendiente arriba), la parte inferior (pendiente abajo) y el centro del disco. En esta última metodología, acorde a la geometría del equipo empleado (20 cm de diámetro), los autores proponen un ajuste del potencial de suministro de aproximadamente $1 \mathrm{~mm}$ por cada unidad porcentual de gradiente de las laderas.

\section{RESULTADOS Y DISCUSIÓN}

El contenido de agua de todos los suelos, al inicio de las mediciones, se mantuvo cercano a capacidad de campo. Para la tensión inicial, $\psi \mathrm{s}=140 \mathrm{~mm}$, se alcanzó el estado de equilibrio dinámico alrededor de los 30 minutos en SA, SFA y SFa, pero en el caso del SaF se alcanzó antes (15 min). En el Cuadro 1 se caracterizan físicamente los suelos y se incluye los valores de materia orgánica de los horizontes superficiales. De acuerdo a la descripción morfológica, todos los suelos presentan uniformidad en sus propiedades, al menos en los dos primeros horizontes. En términos de la estructura superficial, ésta fue de bloques subangulares, salvo el SaF con un grado superficial de grano simple.

Los parámetros de regresión que definen la expresión bilineal (Cuadro 2), se indican gráficamente en la Figura 2.

La tensión pivote, $\psi_{\mathrm{b}}$, mostró (Cuadro 2) una relación directa con la densidad aparente superficial (Da; Cuadro 1). De esta manera, para el SaF, el mayor valor 


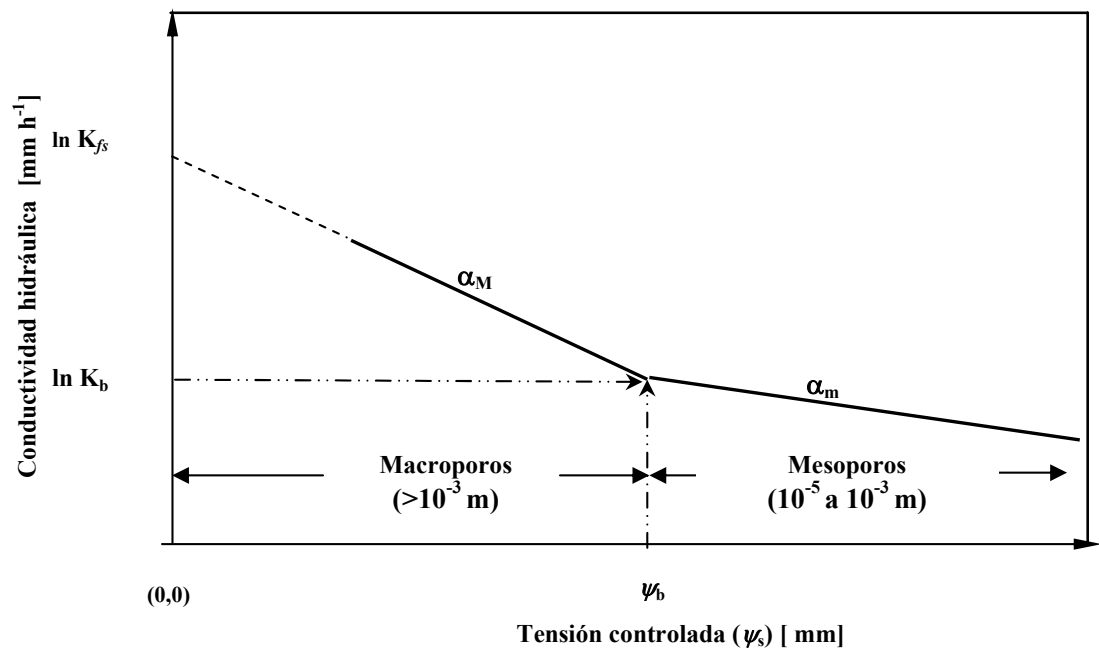

Figura 2. Esquema del modelo de ajuste bilineal de regresión

Figure 2. Outline of adjustment two-line regression model

Cuadro 1. Propiedades físicas y contenidos superficiales de materia orgánica de los suelos.

Table 1. Soils physical properties and organic matter contents (surface horizons).

\begin{tabular}{|c|c|c|c|c|c|c|c|c|c|}
\hline \multirow{2}{*}{\multicolumn{2}{|c|}{ Suelos }} & Arcilla & Limo & Arena & Dp & Da & $\mathbf{W}_{1500}$ & $\mathbf{W}_{33}$ & M.O \\
\hline & & \multicolumn{3}{|c|}{ 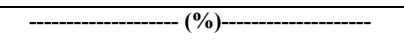 } & \multicolumn{2}{|c|}{--- $\left(\mathrm{Mg} \mathrm{m}^{-3}\right)-$} & \multicolumn{3}{|c|}{ 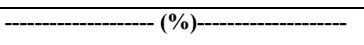 } \\
\hline \multirow{3}{*}{$\mathbf{S}_{\mathbf{A}}$} & $\mathbf{H}_{0-20}$ & $52,45 \pm 2,11$ & $29,12 \pm 0,70$ & $18,43 \pm 2,31$ & $2,61 \pm 0,05$ & $1,30 \pm 0,10$ & $23,16 \pm 0,38$ & $42,07 \pm 0,22$ & $5,38 \pm 0,52$ \\
\hline & $\mathbf{H}_{20-65}$ & $48,04 \pm 2,13$ & $35,28 \pm 0,00$ & $16,68 \pm 2,13$ & $2,63 \pm 0,03$ & $1,32 \pm 0,22$ & $19,50 \pm 0,22$ & $37,07 \pm 0,10$ & \\
\hline & $\mathbf{H}_{65-85}$ & $19,79 \pm 0,88$ & $76,50 \pm 0,38$ & $3,72 \pm 0,50$ & $2,62 \pm 0,03$ & $1,08 \pm 0,06$ & $12,19 \pm 0,07$ & $32,37 \pm 0,04$ & \\
\hline \multirow{4}{*}{$\mathbf{S}_{\mathrm{FA}}$} & $\mathrm{H}_{0-14}$ & $27,93 \pm 0,83$ & $37,14 \pm 1,94$ & $34,93 \pm 1,87$ & $2,64 \pm 0,00$ & $1,40 \pm 0,07$ & $13,14 \pm 0,12$ & $27,85 \pm 0,21$ & $3,12 \pm 0,08$ \\
\hline & $\mathbf{H}_{14-28}$ & $25,62 \pm 0,00$ & $35,07 \pm 1,05$ & $39,31 \pm 1,05$ & $2,63 \pm 0,07$ & $1,70 \pm 0,06$ & $12,84 \pm 0,28$ & $26,18 \pm 0,75$ & \\
\hline & $\mathbf{H}_{28-49}$ & $21,85 \pm 1,04$ & $31,00 \pm 1,03$ & $47,16 \pm 0,00$ & $2,67 \pm 0,04$ & $1,81 \pm 0,04$ & $11,30 \pm 0,22$ & $24,11 \pm 0,65$ & \\
\hline & $\mathbf{H}_{49-80}$ & $19,37 \pm 0,51$ & $32,24 \pm 1,05$ & $48,39 \pm 0,52$ & $2,79 \pm 0,19$ & $1,87 \pm 0,03$ & $11,09 \pm 0,12$ & $25,27 \pm 0,31$ & \\
\hline \multirow{3}{*}{$\mathbf{S}_{\mathbf{F a}}$} & $\mathbf{H}_{0-18}$ & $16,78 \pm 1,27$ & $24,76 \pm 2,41$ & $58,46 \pm 2,37$ & $2,63 \pm 0,00$ & $1,16 \pm 0,00$ & $10,23 \pm 0,09$ & $23,85 \pm 0,41$ & $4,01 \pm 0,76$ \\
\hline & $\mathbf{H}_{18-40}$ & $17,44 \pm 1,02$ & $23,79 \pm 0,00$ & $58,77 \pm 1,03$ & $2,65 \pm 0,00$ & $1,61 \pm 0,00$ & $9,49 \pm 0,07$ & $19,13 \pm 0,12$ & \\
\hline & $\mathbf{H}_{40-70}$ & $16,92 \pm 0,50$ & $25,33 \pm 0,51$ & $57,74 \pm 0,00$ & $2,63 \pm 0,04$ & $1,64 \pm 0,00$ & $8,98 \pm 0,14$ & $18,10 \pm 0,23$ & \\
\hline $\mathbf{S}_{\mathrm{aF}}$ & $\mathbf{H}_{\mathbf{0}-50}$ & $3,43 \pm 0,61$ & $11,79 \pm 2,82$ & $84,60 \pm 3,40$ & $2,57 \pm 0,14$ & $1,67 \pm 0,09$ & $2,73 \pm 0,13$ & $8,28 \pm 0,84$ & $0,91 \pm 0,01$ \\
\hline
\end{tabular}

Dp y Da: densidad de partícula y aparente, respectivamente; W1500 y W33: contenido de agua gravimétrico a 1.500 y $33 \mathrm{kPa}$, respectivamente; M.O: materia orgánica; Hx-x: horizonte genético y profundidad $(\mathrm{cm}) ; \mathrm{S}_{\mathrm{A}}$, $\mathrm{S}_{\mathrm{FA}}, \mathrm{S}_{\mathrm{Fa}} \mathrm{y} \mathrm{S}_{\mathrm{aF}}$ : suelo arcilloso, franco arcilloso, franco arenoso y areno francoso, respectivamente 
Cuadro 2. Parámetros de regresión bilineal entre tensión y conductividad hidráulica de los suelos para cada gradiente de pendiente.

Table 2. Parameters of bilinear approach between tension and hydraulic conductivity for soils at each slope gradient.

\begin{tabular}{|c|c|c|c|c|c|c|}
\hline \multirow{2}{*}{ Suelo } & $\mathbf{P}$ & $\mathbf{K}_{f s}$ & $\psi_{b}$ & $\boldsymbol{\alpha}_{M}$ & $\alpha_{\mathrm{m}}$ & \multirow{2}{*}{$\mathbf{R}_{b}^{2}$} \\
\hline & $(\%)$ & $\left(\mathrm{mm} \mathrm{h}^{-1}\right)$ & (mm) & יב-ב-ביב- & 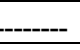 & \\
\hline \multirow{4}{*}{$\mathbf{S}_{\mathbf{A}}$} & 0 & 1,94 & 75 & 0,036 & 0,016 & 0,919 \\
\hline & 15 & 3,00 & 65 & 0,043 & 0,031 & 0,960 \\
\hline & 20 & 2,05 & 75 & 0,038 & 0,022 & 0,937 \\
\hline & 25 & 1,51 & 75 & 0,027 & 0,029 & 0,915 \\
\hline \multirow{4}{*}{$\mathbf{S}_{\mathrm{FA}}$} & 0 & 2,51 & 65 & 0,046 & 0,030 & 0,958 \\
\hline & 15 & 5,95 & 75 & 0,050 & 0,020 & 0,941 \\
\hline & 20 & 3,26 & 65 & 0,041 & 0,017 & 0,890 \\
\hline & 25 & 2,28 & 65 & 0,029 & 0,020 & 0,875 \\
\hline \multirow{4}{*}{$\mathbf{S}_{\mathrm{Fa}}$} & 0 & 11,64 & 55 & 0,080 & 0,016 & 0,873 \\
\hline & 15 & 13,15 & 65 & 0,075 & 0,014 & 0,905 \\
\hline & 20 & 8,03 & 65 & 0,061 & 0,028 & 0,931 \\
\hline & 25 & 3,65 & 65 & 0,052 & 0,025 & 0,873 \\
\hline \multirow{4}{*}{$S_{\mathrm{aF}}$} & 0 & 14,96 & 75 & 0,026 & 0,015 & 0,864 \\
\hline & 15 & 14,57 & 85 & 0,029 & 0,006 & 0,910 \\
\hline & 20 & 11,14 & 75 & 0,027 & 0,007 & 0,805 \\
\hline & 25 & 4,46 & 75 & 0,018 & 0,004 & 0,651 \\
\hline
\end{tabular}

$\mathrm{S}_{\mathrm{A}}, \mathrm{S}_{\mathrm{FA}}, \mathrm{S}_{\mathrm{Fa}}$ y $\mathrm{S}_{\mathrm{aF}}$ : suelo arcilloso, franco arcilloso, franco arenoso y areno francoso, respectivamente. $\mathrm{R}^{2} \mathrm{~b}$ : coeficiente de determinación de ajuste bilineal; $\mathrm{P}$ : gradiente de pendiente $\mathrm{K}_{\mathrm{fs}}$ : conductividad hidráulica saturada; $\psi_{b}$ : tensión pivote; $\alpha \mathrm{M}$ y $\alpha \mathrm{m}$ : gradientes de pendiente de recta en la zona de macroporos $\mathrm{y}$ microporos, respectivamente (Figura 2).

de $\mathrm{Da}\left(1,67 \mathrm{mg} \mathrm{m}^{-3}\right)$ se asocia al mayor valor para $\psi_{\mathrm{b}}$; esto obedece al hecho que presenta un grado de no estructura (grano simple) en todo su perfil, reflejando la comparativamente mayor proporción de macroporos en este suelo. Con respecto a la conductividad hidráulica saturada $(\mathrm{Kfs})$, los valores mayores se encontraron en suelos con clase textural gruesa, independiente de la inclinación. No obstante, para un mismo suelo se observa un incremento anómalo de $\mathrm{Kfs}$ a un gradiente de pendiente de $15 \%$ y una disminución a medida que aumenta este porcentaje (Cuadro 2).

Bodhinayake et al. (2004) observan para suelos inclinados (0 a 20\%) que no existen diferencias significativas en las propiedades hidráulicas ni en la porosidad que conduce agua, estimada in situ con tensioinfiltrómetro, lo cual llevó a Reynolds (2006) a concluir que existiría cierta compensación en los potenciales a medida que se inclina el disco. Sin embargo, al efectuar un ajuste de $0 \mathrm{~mm}$, $14,8 \mathrm{~mm}, 19,6 \mathrm{~mm}$ y $24,2 \mathrm{~mm}$ a los $\psi_{\mathrm{s}}$ pre-establecidos (no se observó flujo superficial al inclinar el infiltrómetro de tensión) para los gradientes de pendiente $0 \%, 15 \%, 20 \%$ y $25 \%$, respectivamente (Casanova et al., 2000a), se aprecia (Figura 3) una disminución más clara de las $\mathrm{Kfs}$ al incrementarse el gradiente de pendiente.

La tendencia resultante del ajuste se explica por las diferencias entre la conductividad hidráulica vertical, dominante en suelos planos, y la 


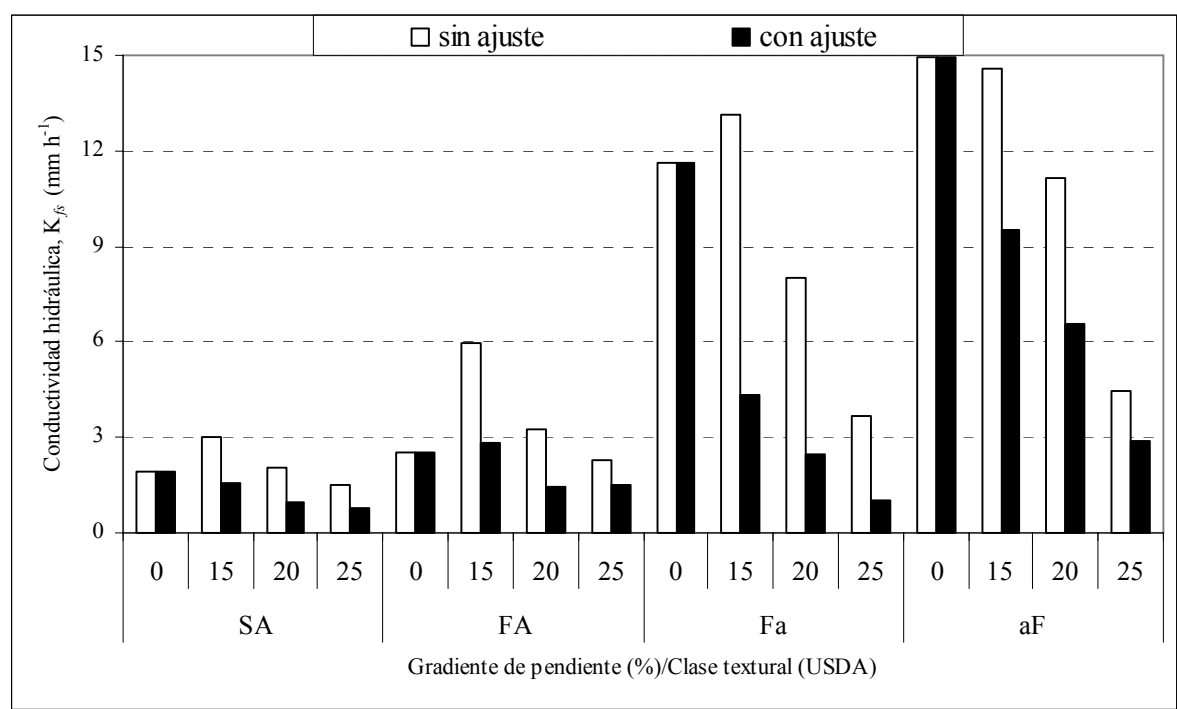

Figura 3. Conductividad hidráulica saturada $\left(\mathrm{K}_{\mathrm{fs}}\right)$ estimada de acuerdo a la textura del suelo y al gradiente de pendiente, con y sin ajuste gravitacional de la tensión controlada con tensioinfiltrómetro.

Figure 3. Saturated hydraulic conductivity $\left(\mathrm{K}_{f s}\right)$ estimated according to soil texture and slope gradient, with and without gravitational adjustment for supply tension head on tension infiltrometer.

conductividad hidráulica horizontal o lateral que comienza a operar por efecto del gradiente de pendiente (Ritsema et al., 1996). Sharma et al. (1983) sugieren que las bajas tasas de infiltración observadas en laderas inclinadas se deben a los periodos cortos de permanencia, ya que la velocidad de flujo superficial es mayor en estas condiciones. Huat et al. (2006) agregan que para un ángulo de pendiente pronunciado la fuerza de gravedad, que actúa en forma descendente durante la infiltración, se resuelve de acuerdo al coseno del ángulo de la pendiente y actúa perpendicularmente a la pendiente de la ladera.

El potencial mátrico, en las mediciones de estos autores, indica que los frentes de mojamiento no son paralelos a la superficie del suelo inclinado. Por lo tanto, la tendencia observada en los datos de esta investigación puede ser producto de la mayor resistencia friccional o viscosa (Hillel, 2004), que se genera al inclinar los monolitos, dado que el agua tiende a avanzar relativamente más adherida a las paredes de los poros al aumentar el gradiente de pendiente. Este efecto es descrito teóricamente por Miyazaki (2006) como una refracción del flujo de agua.

Tanto Bodhinayake et al. (2004) como Casanova et al. (2003) en parcelas inclinadas observaron igualmente una disminución de Kfs al aumentar el gradiente de pendiente, sin embargo, los primeros autores no obtuvieron resultados concluyentes con tensioinfiltrómetro, pero sí al emplear cilindro infiltrómetro.

En concordancia con lo obtenido por algunos autores (Ventrella et al., 2005; Casanova et al., 2000a), al comparar la pendiente de las rectas para cada región de poros, se puede apreciar (Cuadro 2, Figura 4) una notable diferencia entre $\alpha \mathrm{M}$ y $\alpha \mathrm{m}$, salvo en los suelos de textura más 

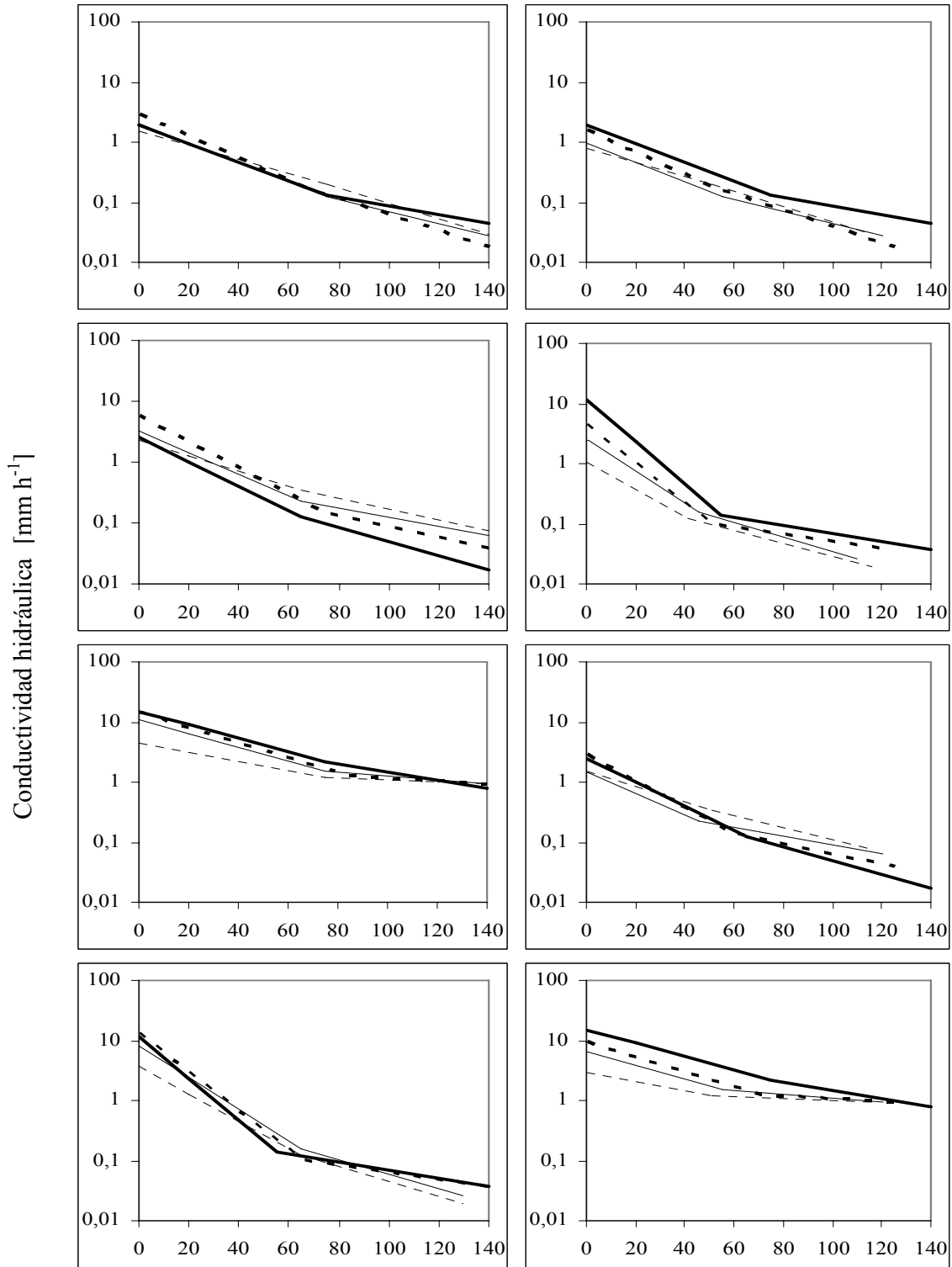

Tensión $[\mathrm{mm}]$

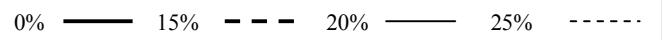

Figura 4. Comparación de la conductividad hidráulica de suelos de textura y gradientes de pendiente diferentes, antes (izquierda) y después (derecha) de un ajuste del potencial controlado afectado por la inclinación.

Figure 4. Comparison between hydraulic conductivity for soils with different textures and slope gradients before (left) and after (right) an adjustment on controlled tension affected by inclination. 
extrema $\left(\mathrm{S}_{\mathrm{A}}\right.$ y $\left.\mathrm{S}_{\mathrm{aF}}\right)$, evidenciándose una tendencia más lineal que bilineal.

Este comportamiento se puede atribuir a que dichos suelos presentarían una relativamente mayor homogeneidad en el tamaño de sus poros que el resto de los suelos, de texturas medias $\left(\mathrm{S}_{\mathrm{Fa}}\right.$ y $\left.\mathrm{S}_{\mathrm{Fa}}\right)$.

\section{CONCLUSIONES}

A diferencia de otras investigaciones que evalúan el efecto del gradiente de pendiente en la conductividad hidráulica saturada $\left(\mathrm{K}_{f s}\right)$ del suelo midiendo en distintas posiciones de las laderas con evidentes cambios en los suelos, la metodología aquí utilizada de inclinar los mismos suelos, permite corroborar que existe una disminución de la magnitud de esta propiedad al incrementarse el gradiente de pendiente, independientemente de la clase textural.

Al operar el tensioinfiltrómetro inclinado y proceder a realizar un ajuste gravitacional a los potenciales controlados, el pivote $\left(\psi_{b}\right)$ disminuyó, obteniéndose conductividades hidráulicas menores a las medidas sin ajuste. Estos resultados obedecerían a las diferencias entre la Kfs vertical (suelos planos) y la Kfs horizontal o lateral que comienza a operar por efecto del gradiente de pendiente; también se puede atribuir a la mayor resistencia friccional o viscosa que se genera al inclinar el suelo, dado que el agua tiende a avanzar en una forma menos libre, lo que es reconocido como una refracción del flujo de agua.

Por otra parte, de acuerdo a los modelos bilineales de regresión, que dan cuenta de dos sistemas porosos (macroporos y mesoporos) para la conductividad hidráulica insaturada $\left(\mathrm{K}_{\psi}\right)$, solo los suelos de clase textural más extrema (arcillosa y areno francosa), presentaron una forma más lineal, debido a la mayor homogeneidad del tamaño de poros que los caracteriza.

Finalmente, se estima que es posible emplear infiltrómetros de disco en laderas inclinadas, al menos hasta un $25 \%$ de gradiente de pendiente, para determinar la conductividad hidráulica de los suelos, si se efectúa la corrección de los potenciales hídricos controlados.

\section{REFRENCIAS}

Angulo-Jaramillo, R., Vandervaere, J., Roulier, S., Thony, J., Gaudet, J., Vauclin, S. 2000. Field measurement of soil surface hydraulic properties by disc and ring infiltrometers. A review and recent developments. Soil and Tillage Research 55, 129.

Ankeny, M., Ahmed, M., Kaspar, T., Horton, R. 1991. Simple field method for determining unsatured hydraulic conductivity. Soil Science Society of American Journal 55, 467 - 470.

Bodner, G., Loiskandl, W., Buchan, G., Kaul, H. 2008. Natural and management-induced dynamics of hydraulic conductivity along a cover-cropped field slope. Geoderma 146, $317-$ 325.

Bodhinayake, W., Cheng Si, B., Noborio, K. 2004. Determination of hydraulic properties in sloping landscapes from tension and double-ring infiltrometers. Vadose Zone Journal 3, 964-970.

Bradford, J.M., Huang, C. 1992. Mechanisms of crust formation: physical components. In: M. E. Sumner and B. A. Stewart.(eds.). Advances in Soil Science. Soil crusting: physical and chemical processes. Boca Raton. Lewis Publishers, pp: $55-$ 72.

Casanova, M., Messing, I., Joel, A. 2000a. Influence of aspect and slope gradient on hydraulic conductivity measured by tension infiltrometer. Hydrological Processes 14(1), 155 164.

Casanova, M., Benavides, C., Poblete, I., Joel., A., Seguel, O. 2000b. Tensioinfiltómetro: Conductividad hidráulica de un suelo aluvial acondicionado con materiales orgánicos. In: Aragón et al. (Eds.): Avances en Ingeniería Agrícola. Facultad de Agronomía, Universidad de Buenos Aires, Argentina, pp. 372-377. 
Casanova, M., Seguel, O., Joel, A., Messing, I., Luzio, W., Vera, W. 2003. Funciones de pedotransferencia para conductividad hidráulica en laderas de secano. Revista de la Ciencia del Suelo y Nutrición Vegetal 3, 42 - 48.

Chahinian, N., Voltz, M., Moussa, R., Trotoux G. 2006. Assessing the impact of the hydraulic properties of a crusted soil on overland flow modeling at the field scale. Hydrological Processes 20(8), 1701-1722.

CIREN, 1996. Estudio Agrológico Región Metropolitana. Descripciones de suelos, materiales y símbolos. Centro de Información de Recursos Naturales. Publicación $\mathrm{N}^{\circ} 115$. Santiago. 463 p.

Cook, F.J. 2006. Unsaturated hydraulic conductivity: Laboratory tension infiltrometer. In: Carter, M.R., E.G. Gregorich (Eds.): Soil Sampling and Methods of Analysis. (2nd Ed.). Canadian Society of Soil Science. CRC Press, Taylor \& Francis Group, pp: 1070-1082.

D'Odorico, P., Porporato, A. 2006. Soil moisture dynamics in water-limited ecosystems. In: D’Odorico, P., Porporato, A.( Eds.): Dryland Ecohydrology. Springer, New York, pp: 31-46.

FAO, 2000. Land resource potential and constraints at regional and country levels. Land and Water Development Division. Food and Agriculture Organization of the United Nations. World Soil Resources Reports 90. Rome. 122 p.

Gee, R., Bauder, P. 1986. Particle size. In: Klute, A. (Ed.): Methods of soil analysis. Part 1. (2nd Ed.). A.S.A. and S.S.S.A., Agronomy Monograph $\mathrm{N}^{\circ}$. Madison, WI. USA, pp: 443461.

Hillel, D. 2004. Introduction to Environmental Soil Physics. Elsevier Academic Press. San Diego. CA. USA. 494 p.

Hu, W., Shao, M., Wang, Q., Fan, J., Horton, R. 2009. Temporal changes of soil hydraulic properties under different land uses. Geoderma 149(3-4), 355-366.

Huat, B.B.K., Ali, F.H.J., Low, T.H. 2006. Water infiltration characteristics of unsaturated soil slope and its effect on suction and stability. Geotechnical and Geological Engineering 24, 1293-1306.
Hussen, A.A., Warrick, A.W. 1993. Alternative analysis of hydraulic data from the disc infiltrometers. Water Resources Research 29, 4103- 4108 .

Janeau, J.L., Bricquet, J.P., Planchon, O., Valentin, C. 2003. Soil crusting and infiltration on steep slopes in northern Thailand. European Journal of Soil Science 54, 543-553.

Keng, J.C., Lin, C.S. 1982. A two-line approximation of hydraulic conductivity for structured soils. Canadian Agricultural Engineering 24 (2), 77-80.

Klute, A. 1986. Methods of Soil Analysis. Part 1. (2nd Ed.). A.S.A. and S.S.S.A. Agronomy Monograph $\mathrm{N}^{\circ}$. Madison, WI. USA. $1188 \mathrm{p}$.

López, I.F., Lambert, M.G., Mackay, A.D., Valentine, I. 2003. The influence of topography and pasture management on soil characteristics and herbage accumulation in hill pasture in the North Island of New Zealand. Plant and Soil 255, 421-434.

Logsdon, S.D., Jaynes, D.B. 1993. Methodology for determining hydraulic conductivity with tension infiltrometers. Soil Science Society of American Journal 57, 1426-1431.

Luxmoore, R.J. 1981. Micro-, meso-, and macroporosity of soil. Soil Science Society of American Journal 45, 671-672.

Mertens, J., Jacques, D., Vanderborght, J., Feyen, J. 2002. Characterization of the fieldsaturated hydraulic conductivity on a hillslope: in situ single ring pressure infiltrometer measurements. Journal of Hydrology 263, 217 219.

Messing, I., Jarvis, N.J. 1993. Temporal variation in the hydraulic conductivity of a tilled clay soil as measured by tension infiltrometer. Journal of Soil Science 44, 11- 24.

Miyazaki, T. 2006. Water flow in soils. (2nd Ed.). University of Tokyo, Japan. CRC Press, Taylor \& Francis Group. 402 p.

Reynolds, W. 2006. Unsatured hydraulic properties: Field tension infiltrometer. In: Carter, M.R., E.G., Gregorich (Eds.): Soil Sampling and Methods of Analysis. (2nd Ed.). Canadian Society of Soil Science. CRC Press, Taylor \& Francis Group, pp: 1101-1121. 
Reynolds, W., Elrick, E., Youngs, E., Booltink, H., Bouma, J. 2002. Saturated and field saturated water flow parameters. In: Dane J. and Topp G. (Eds.) Methods of Soil Analysis. Part 4. Physical Methods. ASA and SSSA, Madison, WI, pp: 797 -816 .

Reynolds, W. 1993. Unsaturated hydraulic conductivity: Field measurements. In: Carter, M. (Ed.) Soil Sampling and Methods of Analysis. Canadian Society of Soil Science. Lewis Publishers, pp: 633 - 644.

Ridolfi, L., D'Odorico, P., Porporato, A., Rodriguez-Iturbe, I. 2003. Stochastic soil moisture dynamics along a hillslope. Journal of Hydrology 272, 264-275.

Ritsema, C. J., Oostindie, K., Stolte, J. 1996. Evaluation of vertical and lateral flow through agricultural loessial hillslopes using a twodimensional computer simulation model. Hydrological Processes 10, 1091-1105.

Sadzawka, A., Flores, H., Carrasco, M.A., Grez, R., Mora, M.L., Neaman, A., Demanet, R. 2007. Métodos de análisis de lodos y de suelos. Comisión de Normalización y Acreditación, Sociedad Chilena de la Ciencia del Suelo. 105 p.

Sharma, K., Singh, H., Pareek, O. 1983. Rainwater infiltration into a bare loamy sand. Hydrological Science Journal 28, 417-424.

Sherrod, P.H.. 2005. NLREG: nonlinear regression analysis program (version 6.3). Brentwood, TN, USA.
Strudley, M.W., Green, T.R., Ascough, J.C. 2008. Tillage effects on soil hydraulic properties in space and time State of the science. Soil and Tillage Research 99, 4-48.

Sullivan, M., Warwick, J.J., Tyler, S.W. 1996. Quantifying and delineating spatial variations of surface infiltration in a small watershed. Journal of Hydrology 181, 149-168.

Ventrella, D., Losavio, N., Vonella, A.V., Leij, F.J. 2005. Estimating hydraulic conductivity of a fine-textured soil using tensioninfiltrometry. Geoderma 124, 267-277.

Verbist, K., Baetens, J., Cornelis, W.M., Gabriels, D., Torres, C., Soto, G. 2009. Hydraulic conductivity as influenced by stoniness in degraded drylands of Chile. Soil Science Society of American Journal 73, 471-484.

Walker, C., Lin, H.S., Fritton, D. 2006. Is the tension beneath a tension infiltrometer what we think it is?. Vadose Zone Journal 5, 860-866.

Wooding, R.A. 1968. Steady infiltration from a shallow circular pond. Water Resources Research 4, 1259-1273.

Zaslavsky, D., Sinai, G. 1981. Surface hydrology: II. Distribution of raindrops. Journal of Hydraulic Engineering Division ASCE 107(HY1), 17-35 